\title{
Usefulness of 18-FDG PET/CT in Detecting Malignancy in Intraductal Papillary Mucinous Neoplasms of the Pancreas
}

\author{
YO-ICHI YAMASHITA, HIROHISA OKABE, HIROMITSU HAYASHI, KATSUNORI IMAI, \\ SHIGEKI NAKAGAWA, YOSUKE NAKAO, TOSHIHIKO YUSA, RUMI ITOYAMA, TAKANOBU YAMA, \\ NAOKI UMESAKI, KOTA ARIMA, TATSUNORI MIYATA, AKIRA CHIKAMOTO and HIDEO BABA
}

Department of Gastroenterological Surgery, Graduate School of Life Sciences, Kumamoto University, Kumamoto, Japan

\begin{abstract}
Background/Aim: Identifying malignancy in intraductal papillary mucinous neoplasm (IPMN) of the pancreas remains challenging. This study aimed to evaluate the usefulness of 18-fluorodeoxyglucose positron emission tomography/computed tomography (18-FDG PET/CT) in distinguishing malignant from benign IPMN of the pancreas. Patients and Methods: The cases of 79 patients with IPMN of the pancreas who underwent surgical resections between 1996 and 2016 at our Institution were enrolled in the present retrospective analysis of predictors for malignancy in IPMN of the pancreas. 18-FDG PET/CT evaluations were performed in 38 patients, and the usefulness of 18-FDG $P E T / C T$ for detecting malignancy in IPMN of the pancreas was evaluated. Results: Three factors were significantly related to malignancy in IPMN; the diameter of the main pancreatic duct, the serum value of carcinoembryonic antigen (CEA), and the neutrophil-to-lymphocyte ratio (NLR). 18-FDG PET accumulation was significantly related to malignancy in IPMN; sensitivity 0.82 and specificity 0.71 . Independent predictors for malignancy in IPMN were as follows: 18-FDG PET accumulation, CEA >1.0 $\mathrm{ng} / \mathrm{ml}$, and NLR >2.63. Conclusion: 18-FDG PET accumulation is a potent new marker for distinguishing malignant from benign IPMNs of the pancreas.
\end{abstract}

Since the first description by Ohashi and colleagues in 1982 (1), intraductal papillary mucinous neoplasm (IPMN) of the pancreas has been widely discussed. IPMN was defined by the World Health Organization (WHO) in 1996 as an intraductal

Correspondence to: Yo-ichi Yamashita, MD, Ph.D., F.A.C.S., Department of Gastroenterological Surgery, Graduate School of Life Sciences, Kumamoto University, 1-1-1 Honjyo, Chuo-ku, Kumamoto 860-8556, Japan. Tel: +81 963735211, Fax: +81 963714378, e-mail: y-yama@kumamoto-u.ac.jp

Key Words: 18-FDG PET/CT, IPMN of the pancreas, predictors, IPMCa, neutrophil-to-lymphocyte ration (NLR). mucin-producing neoplasm with tall columnar, mucincontaining epithelium, with or without papillary projections, involving the main pancreatic duct and/or major side branches and lacking ovarian stroma characteristics of mucinous cystic neoplasms $(2,3)$. It is now well established that IPMN represents a pancreatic precancerous lesion, following a stepwise pattern of malignant transformation, similar to that of adenomatous polyp in colon cancer (4). Differentiating between benign/malignant IPMN is thus important, but the question of how to do so remains open and crucial.

The surgical resection of a premalignant/malignant IPMN offers a window of opportunity for potential cure, but at the cost of considerable morbidity (up to $40 \%$ ) and mortality $(1-5 \%)$, even at experienced centers $(5,6)$. To address this conundrum of under- $v s$. overtreatment, the International Association of Pancreatology published the International Consensus Guidelines (ICGs) for surgical indications in 2006 (7), revised them in 2012 (8) and again in 2017 (9). Some retrospective studies validated the safety of these recommendations, and all concluded that the guidelines' specificity in distinguishing benign from malignant IPMN was low $(10,11)$, resulting in $75-85 \%$ of the surgical procedures being potentially unnecessary.

The 2017 revised ICGs describe, "high-risk stigmata" and "worrisome features" consisting of several clinical factors such as obstructive jaundice, the diameter of the enhancing mural nodule, the main pancreatic duct (MPD) diameter, the cyst diameter, an increased level of carbohydrate antigen 199 (CA19-9), and more (9). The simultaneous use of several clinical factors might help improve the sensitivity and specificity in differentiating between benign/malignant diagnosis for IPMNs, but the existence of many clinical factors in the current algorism means that there has been no definite imaging tool for the accurate diagnosis of benign/malignant IPMN.

The use of 2-[18F]-fluoro-2 deoxy-D-glucose positron emission tomography/computed tomography (18-FDG $\mathrm{PET} / \mathrm{CT}$ ) for oncologic imaging is well established and widely accepted for many malignancies, and it is becoming 
Table I. Predictors for IPMCa.

\begin{tabular}{lccc}
\hline Variables & IPMA $(\mathrm{n}=49)$ & IPMCa $(\mathrm{n}=30)$ & $p$-Value \\
\hline Age & $67 \pm 8$ & $70 \pm 9$ & 0.2225 \\
Male/Female & $35 / 14$ & $20 / 10$ & 0.6561 \\
Cyst diameter $(\mathrm{mm})$ & $34 \pm 14$ & $36 \pm 24$ & 0.5847 \\
MPD diameter $(\mathrm{mm})$ & $7 \pm 5$ & $10 \pm 7$ & 0.0105 \\
Mural nodule $(+)$ & $21(43 \%)$ & $17(56 \%)$ & 0.1989 \\
CEA $(\mathrm{ng} / \mathrm{ml})$ & $1.9 \pm 1.5$ & $2.6 \pm 1.9$ & 0.0307 \\
CA19-9 $(\mathrm{U} / \mathrm{ml})$ & $34.1 \pm 96.6$ & $80.0 \pm 198.2$ & 0.1724 \\
NLR & $2.0 \pm 0.7$ & $2.6 \pm 0.9$ & 0.0046 \\
\hline
\end{tabular}

IPMCa: Intraductal papillary mucinous carcinoma; MPD: main pancreatic duct; CEA: carcinoembryonic antigen; CA19-9: carbohydrate antigen 19-9; NLR: neutrophil-to-lymphocyte ratio.

an increasingly important imaging modality (12). The potential use of 18-FDG PET/CT for differentiating between benign/malignant IPMNs has been discussed (13-16), but the sample sizes of these studies were too small, and thus the role of 18-FDG PET/CT in the diagnosis of IPMN has not been elucidated. The usefulness of 18 -FDG PET/CT in detecting malignancy in IPMN of the pancreas should be validated using several surgical cohorts.

We herein present a retrospective analysis of the efficacy of 18-FDG PET/CT for differentiating between benign/ malignant IPMNs compared to the efficacy of the clinical variables listed in the ICGs.

\section{Patients and Methods}

Patients. Between January 1996 and December 2016, 79 patients diagnosed with IPMN of the pancreas who underwent curative pancreatic resections at the Department of Gastroenterological Surgery, Kumamoto University were enrolled in this study. Of these patients, $49(62 \%)$ were pathologically diagnosed as having intraductal papillary mucinous adenoma (IPMA) and the other 30 patients $(38 \%)$ were diagnosed as having intraductal papillary mucinous carcinoma (IPMCa). Each patient provided written informed consent before surgery.

Tumors were preoperatively evaluated by diagnostic imaging modalities, including abdominal ultrasonography, CT, MRI, endoscopic ultrasonography (EUS), and 18-FDG PET/CT. This study was approved by the Human Ethics Review Committee of the Graduate School of Medicine, Kumamoto University (No. 1291).

Predictors for malignancy in IPMN of the pancreas. According to the revised ICGs for the management of IPMN or previous reports, we analyzed eight factors as predictors for malignancy in IPMN: patient age, male/female, cyst diameter, MPD diameter, the presence of mural nodules, the serum value of CEA, the serum value of CA19-9, and the neutrophil-to-lymphocyte ratio (NLR) (17).

18-FDG PET/CT. In 38 patients, 18-FDG PET/CT was performed by injecting $10-12 \mathrm{mCi}$ of $18 \mathrm{~F}-\mathrm{FDG}$ after an overnight fast. The patients' blood glucose level was measured just before the tracer administration and was confirmed as within $140 \mathrm{mg} / \mathrm{dl}$. For the quantitative analysis, the maximum of the standardized uptake value (SUVmax) was calculated at the sites of suspected tumor foci on CT scans. The positive uptake of FDG was defined as a focal uptake with an SUVmax $\geq 1.3$.

Statistical analysis. Continuous variables are expressed as the mean \pm standard deviation and were compared using Student's $t$-test. Categorical variables were compared using the $\chi^{2}$ test. The optimal cutoff value of continuous variables in predictors of malignancy in IPMN was determined by a receiver operating characteristics (ROC) curve analysis. Variables at $p<0.2$ in a univariate analysis were subjected to a stepwise logistic regression analysis to identify independent predictors of malignancy in IPMN. All analyses were performed with JMP Pro 13.0.0 software (SAS, Cary, NC). $p$-Values $<0.05$ were considered significant.

\section{Results}

Predictors of malignancy in IPMN of the pancreas. Table I summarizes the comparisons of the eight factors between the IPMA $(n=49)$ and IPMCa $(n=30)$ groups. The mean MPD diameter was significantly larger in the IPMCa group compared to the IPMA group $(10 \pm 7 v s .7 \pm 5 \mathrm{~mm}, p=0.0105)$. The mean serum value of CEA was significantly higher in the IPMCa group compared to the IPMA group (2.6 $\pm 1.9 \mathrm{vs}$. $1.9 \pm 1.5 \mathrm{ng} / \mathrm{ml}, p=0.0307)$. The mean serum NLR was significantly higher in the IPMCa group than that in the IPMA group $(2.6 \pm 0.9$ vs. $2.0 \pm 0.7, p=0.0046)$. Although the difference was not significant, the IPMCa group's positive rate of the presence of mural nodule ( $56 v s .43 \%, p=0.1989)$, and the mean serum CA19-9 value $(80.0 \pm 198.2$ vs. $34.1 \pm 96.6$ $\mathrm{U} / \mathrm{ml}, p=0.1724)$ were higher than those in the IPMA group.

The ROC curves of the MPD diameter, CEA, NLR, and CA19-9 are provided in Figure 1. The optimal cutoff values and the area under the curve (AUC) for the prediction of malignancy in IPMN were as follows: MPD diameter 4.0 $\mathrm{mm}$ (AUC 0.67), CEA $1.0 \mathrm{ng} / \mathrm{ml}$ (AUC 0.63), NLR 2.63 (AUC 0.68), and CA19-9 13.5 (AUC 0.64).

Usefulness of 18-FDG PET in detecting malignancy in IPMN of the pancreas. Typical IPMN cases with 18-FDG PET/CT are presented in Figures 2 and 3 . Figure 2 presents the case of an 84-year-old male who was finally diagnosed as having IPMA. An enhanced CT scan revealed a cyst (20 mm diameter) at the uncus of the pancreas with MPD dilatation $(11 \mathrm{~mm}$ ) (Figure 2A). 18-FDG PET/CT revealed no accumulation of $18 \mathrm{~F}-\mathrm{FDG}$ on this cyst (Figure $2 \mathrm{~B}$ ). EUS revealed a mural nodule $(3 \mathrm{~mm}$ diameter) in this pancreatic cyst. The patient underwent a pancreaticoduodenectomy (PD) and was pathologically diagnosed as having IPMA (Figure 1C).

Figure 3 presents the case of a 67-year-old female who was finally diagnosed as having IPMCa. An enhanced CT scan revealed a cyst ( $30 \mathrm{~mm}$ diameter) at the uncus of the pancreas with MPD dilatation (13 mm) (Figure 3A). 18-FDG 


\section{MPD diameter}

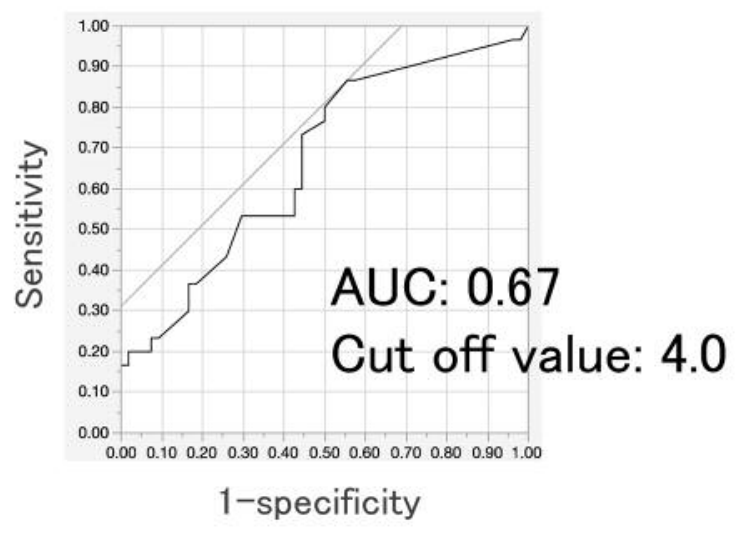

NLR

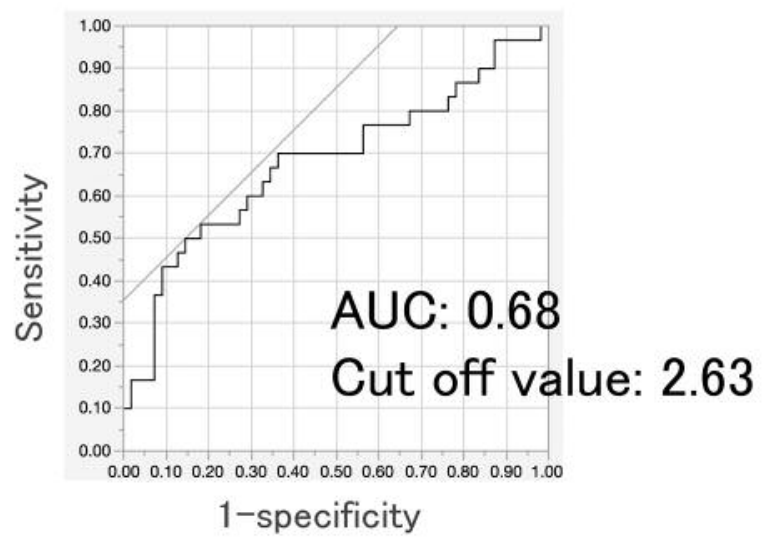

CEA

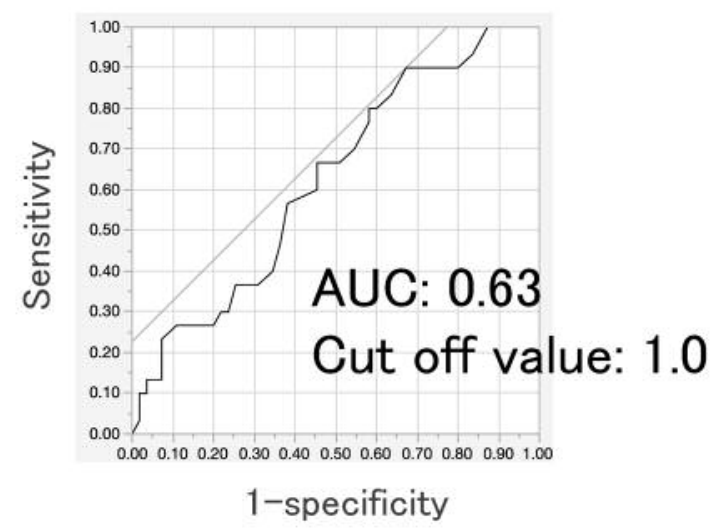

CA19-9

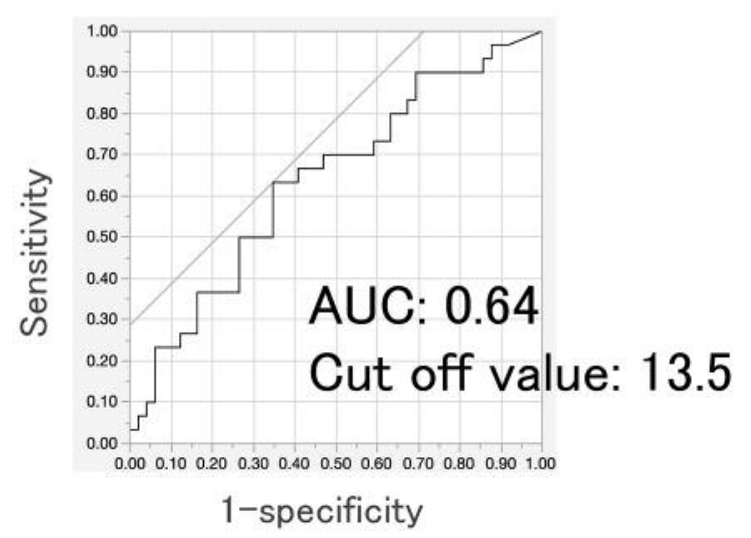

Figure 1. ROC curves of the MPD diameter, serum CEA, the NLR, and serum CA19-9. The optimal cutoff values and areas under the curve (AUC) for the prediction of malignant IPMN were as follows: MPD diameter $4.0 \mathrm{~mm}$ (AUC 0.67), CEA $1.0 \mathrm{ng} / \mathrm{ml}$ (AUC 0.63), NLR 2.63 (AUC 0.68), and CA19-9 13.5 U/ml (AUC 0.64).

PET/CT revealed the accumulation on this cyst (SUVmax 2.8) (Figure 3B). EUS revealed the enhanced mural nodule (16 $\mathrm{mm}$ diameter) in this pancreatic cyst. The patient underwent a PD, and was pathologically diagnosed as having IPMCa with neural invasion (ne3) (Figure 3C).

Among the 38 patients who underwent 18-FDG PET/CT, the $18 \mathrm{~F}-\mathrm{FDG}$ accumulation was recognized in 20 patients $(53 \%)$. The 18F-FDG accumulation was significantly correlated to the IPMCa $(p=0.0007)$, with sensitivity 0.82 and specificity 0.71 , respectively.

Independent predictors of malignancy in IPMN of the pancreas. The results of our stepwise logistic regression analysis for the prediction of IPMCa are summarized in Table II. Three independent predictors of IPMCa in our cohort were identified: $18 \mathrm{~F}-\mathrm{FDG}$ accumulation (odds ratio
$[\mathrm{OR}]=50.7, \quad p=0.0008), \quad \mathrm{CEA}>1.0 \mathrm{ng} / \mathrm{ml} \quad(\mathrm{OR}=18.0$, $p=0.0239)$, and NLR $>2.63(\mathrm{OR}=14.3, p=0.0255)$.

\section{Discussion}

$\mathrm{PET} / \mathrm{CT}$ is a diagnostic tool that enables the evaluation of tissue glucose metabolism abnormalities, and 18F-FDG is the most widely used and currently preferred radiotracer. The use of 18F-FDG PET/CT enables the differentiation between normal and tumor tissue on the basis that neoplastic cells have higher glucose metabolic activity $(18,19)$. The use of 18F-FDG PET/CT for oncological imaging is well established and widely accepted for many types of malignancies, and we have reported the clinical usefulness of 18-FDG PET/CT in assessing the malignant potential of some hepatobiliary pancreatic tumors (20-22). 

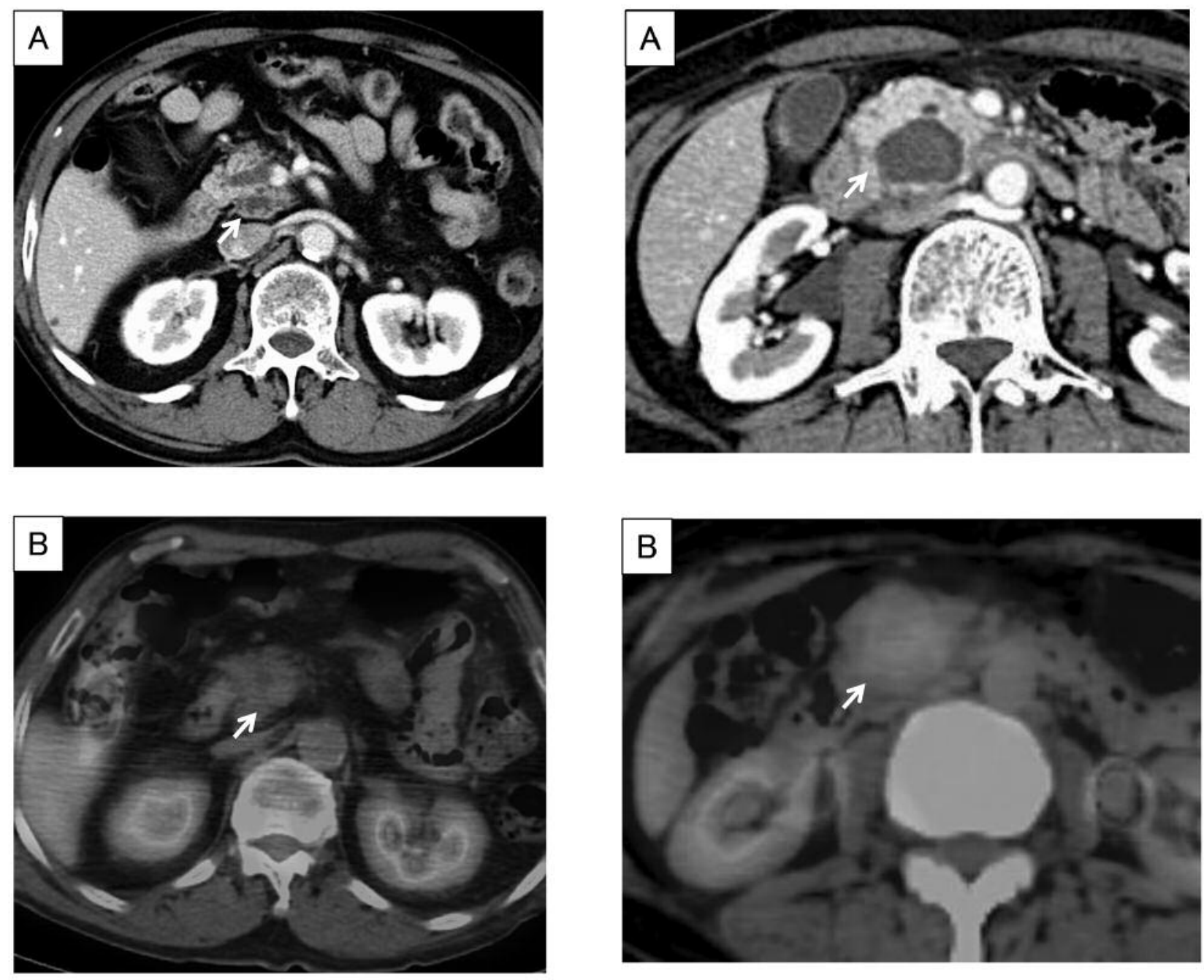

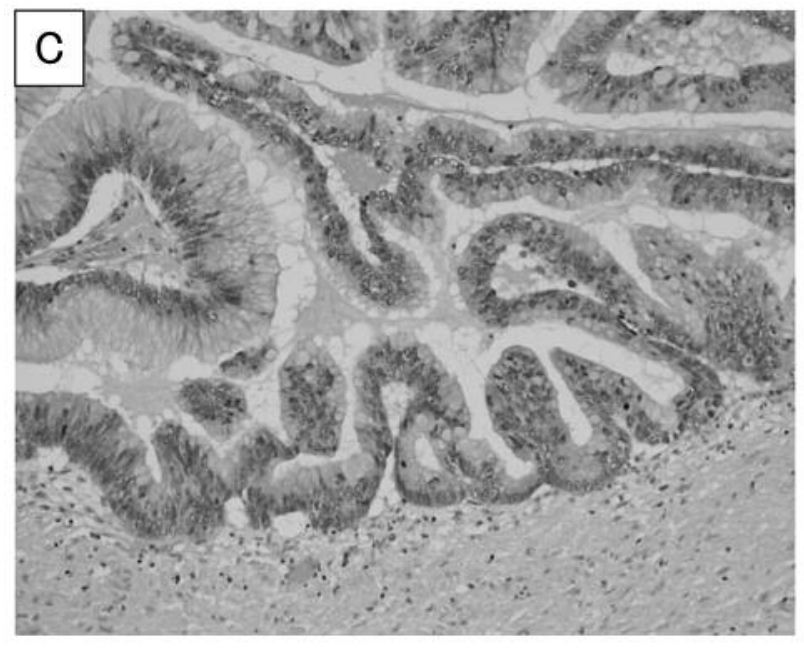

Figure 2. A: Enhanced CT scan revealed the cyst (23 mm diameter) at the uncus of the pancreas (white arrow) with MPD dilatation (11 $\mathrm{mm}$ ). B: 18-FDG PET/CT revealed no accumulation of $18 F-F D G$ on this cyst (white arrow). C: Pathological diagnosis was IPMA.

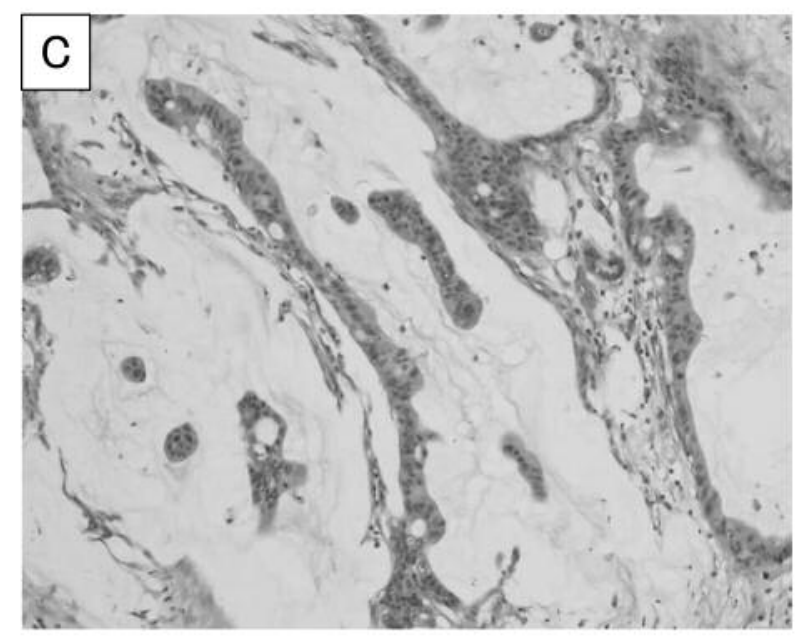

Figure 3. A: Enhanced CT scan revealed the cyst (30 mm diameter) at the uncus of the pancreas (white arrow) with MPD dilatation $(13 \mathrm{~mm})$. B: 18-FDG PET/CT revealed the accumulation on this cyst (SUVmax 2.8) (white arrow). C: Pathological diagnosis was IPMCa. 
The possible use of 18F-FDG PET/CT for detecting malignancy in IPMN of the pancreas has not been widely investigated, but two reports summarized several studies and emphasized its clinical usefulness in differentiating between benign/malignant diagnoses for IPMN $(13,23)$. Bertagna et al. reviewed 60 reports and evaluated the use of 18F-FDG PET/CT for detecting malignancy in IPMN (13). They determined that the pooled sensitivity and specificity of $18 \mathrm{~F}-\mathrm{FDG}$ PET/CT in differentiating between benign/malignant diagnoses for IPMN were 0.88 and 0.98 , respectively. According to these high sensitivity and specificity values, Bertagna et al. concluded that 18F-FDG PET/CT can be expected to lead to an improvement over conventional imaging.

Sultana et al. reported a systematic review and metaanalysis concerning the best way to identify malignant transformation within IPMN of the pancreas (23). They concluded that the best way to detect malignancy of IPMN is the combination of elevated CA19-9 levels and the presence of mural nodule. The sensitivity and specificity of this combination in detecting the malignancy of IPMN were 0.743 and 0.907 , respectively; however, in their own report, the sensitivity and specificity of 18F-FDG PET/CT in detecting the malignancy of IPMN were higher at 0.968 and 0.911 , respectively. They recognized that $18 \mathrm{~F}-\mathrm{FDG}$ PET/CT has promise, but they mentioned that it requires further evaluation by larger studies with improved quality.

The International Association of Pancreatology published ICGs for the surgical indication of IPMN of the pancreas in 2006 (7) and 2012 (8). Roch et al. reported that malignant stratification based on the 2012 ICGs alone was characterized by 0.92 sensitivity and 0.27 specificity (16). This low specificity of the 2012 ICGs in distinguishing malignant from benign IPMNs was widely reported $(11,16)$, resulting in 75 $85 \%$ potentially unnecessary surgical procedures. Because of the aging population and the low rate of the malignant transformation of IPMN (1.4\%-6.9\% per year) $(24,25)$, careful surgical indications for borderline IPMN are needed.

The 2017 revised ICGs (9) newly adopted the size limitation of enhancing mural nodule $(\geq 5 \mathrm{~mm})$ in the high-risk stigmata, and regarding worrisome features, the ICGs adopted three new factors (lymphadenopathy, increased serum level of CA19-9, and cyst growth rate $\geq 5 \mathrm{~mm} / 2$ years) and excluded one factor (non-enhancing mural nodule). The establishment of the precise clinical usefulness of the 2017 revised ICGs for differentiating between benign/malignant diagnoses for IPMN awaits large cohort studies. However, we speculate that the actual evaluation of "enhancement" of a mural nodule is difficult, especially in small nodules $(\leq 10 \mathrm{~mm})$, without the use of EUS (26). Table III summarizes the sensitivity and specificity for predicting IPMCa of five factors described in the 2017 revised ICGs and 18F-FDG PET/CT in our series. Compared to other imaging factors, relatively high sensitivity (0.82) and specificity (0.71) of $18 \mathrm{~F}-\mathrm{FDG}$ PET/CT were
Table II. Independent predictors for IPMCa.

\begin{tabular}{lccc}
\hline Variables & Odds ratio & $95 \% \mathrm{CI}$ & $p$-Value \\
\hline 18F-FDG accumulation (+) & 50.7 & $2.17-1179.55$ & 0.0008 \\
CEA $>1.0 \mathrm{ng} / \mathrm{ml}$ & 18.0 & $1.05-310.8$ & 0.0239 \\
NLR $>2.63$ & 14.3 & $1.01-211.71$ & 0.0255 \\
Mural nodule (+) & 1.85 & $0.23-14.95$ & 0.5568 \\
MPD $>4.0 \mathrm{~mm}$ & 1.71 & $0.21-14.95$ & 0.6135 \\
CA19-9 $>13.5 \mathrm{U} / \mathrm{ml}$ & 3.89 & $0.53-28.48$ & 0.3759 \\
\hline
\end{tabular}

IPMCa: Intraductal papillary mucinous carcinoma; CI: confidence interval; 18F-FDG: 2-[18F]-fluoro-2 deoxy-D-glucose; CEA: carcinoembryonic antigen; NLR: neutrophil-to-lymphocyte ratio; MPD: main pancreatic duct; CA19-9: carbohydrate antigen 19-9.

Table III. Summary of sensitivity and specificity in predicting IPMCa.

\begin{tabular}{lcc}
\hline Variables & Sensitivity & Specificity \\
\hline 18F-FDG accumulation (+) & 0.82 & 0.71 \\
Mural nodule (+) & 0.57 & 0.57 \\
MPD diameter $\geq 10 \mathrm{~mm}$ & 0.43 & 0.71 \\
MPD diameter $5-9 \mathrm{~mm}$ & 0.77 & 0.50 \\
Cyst diameter $\geq 30 \mathrm{~mm}$ & 0.60 & 0.42 \\
CA19-9 $>37 \mathrm{U} / \mathrm{ml}$ & 0.27 & 0.86 \\
\hline
\end{tabular}

IPMCa: Intraductal papillary mucinous carcinoma; 18F-FDG: 2-[18F]fluoro-2 deoxy-D-glucose; MPD: main pancreatic duct; CA19-9: carbohydrate antigen 19-9.

recognized. The high specificity (0.86) of CA19-9 >37 U/ml (newly adopted in the 2017 revised ICGs) for predicting IPMCa was also confirmed in our series, which will thus lead to an improvement of specificity for differentiating between benign/malignant diagnoses for IPMN.

Because of the high accuracy of 18F-FDG PET/CT in differentiating between benign/malignant IPMNs, we recommend that $18 \mathrm{~F}-\mathrm{FDG} \mathrm{PET} / \mathrm{CT}$ should be involved in the algorithm for the management of IPMN in future ICGs. 18FFDG PET/CT can improve the specificity of the preoperative workup for the diagnosis of malignant IPMN. This can prevent potentially unnecessary pancreatic surgeries, especially in elderly patients. 18F-FDG PET/contrastenhanced $\mathrm{CT}$ is a non-invasive modality that can evaluate the 18F-FDG uptake in addition to the conventional high-risk findings, and its use can reduce the number of outpatients' visits (15). However, the routine application of 18F-FDG $\mathrm{PET} / \mathrm{CT}$ for IPMN diagnosis has several problems such as exposure to radioactive elements and cost-effectiveness, especially in IPMN follow-ups. The optimal application of 18F-FDG PET/CT in the algorithm for the management of IPMN should be discussed further. 
Regarding the limitations of the present study, we note that it was a single-institution, retrospective analysis of a small patient number. The optimal cutoff of SUVmax should be determined for predicting malignant IPMN by a larger cohort study. As in our earlier study (17), the clinical usefulness of the NLR in differentiating between benign/malignant IPMN was also confirmed in this study. However, the cutoff value of the NLR differed (2.074 vs. 2.63), and a larger cohort study should be conducted to confirm the clinical usefulness of the NLR in the diagnosis of malignant IPMN.

In conclusion, 18-FDG PET/CT accumulation is a potent new marker for distinguishing malignant from benign IPMNs of the pancreas. This would reduce potentially unnecessary pancreatic surgeries for benign IPMN of the pancreas.

\section{Conflicts of Interest}

The Authors have no conflicts of interest to declare regarding this study.

\section{Authors' Contributions}

Study conception and design: Yamashita; Acquisition of data: Yamashita, Okabe, Hayashi, Imai, Nakagawa, Nakao, Yusa, Itoyama, Yamao, Umesaki, Arima, Miyata, Chikamoto; Analysis and interpretation of data: Yamashita, Okabe; Drafting of manuscript: Yamashita, Hyashi; Critical revision: Baba.

\section{References}

1 Ohashi K, Mayakami F, Maruyama M, Takekoshi T, Ohta H and Ohashi I: Four cases of mucous secreting pancreatic cancer. Prog Dig Endosc 20: 348-351,1982.

2 Kloppel G, Solcia E, Longnnecker DC, Capella C and Sobin LH: Histological typing of tumors of the exocrine pancreas. 2nd ed. Berlin: Springer, 1996.

3 Sakorafas GH, Smyrniotis V, Reid-Lombardo KM and Sarr MG: Primary pancreatic cystic neoplasms of the pancreas revisited. Part IV: rare cystic neoplasms. Surg Oncol 21: 153-163, 2012. PMID: 21816607. DOI: 10.1016/j.suronc.2011.06.007

4 Scarlett CJ, Salisbury EL, Biankin AV and Kench J: Precursor lesions in pancreatic cancer: morphological and molecular pathology. Pathology 43: 183-200, 2011. PMID: 21436628. DOI: 10.1097/PAT.0b013e3283445e3a

5 Cameron JL and He J: Two thousand consecutive pancreaticoduodenectomies. J Am Coll Surg 220: 530-536, 2015.

6 Parikh P, Shiloach M, Cohen ME, Bilimoria KY, Ko CY, Hall BL and Pitt HA: Pancreatectomy risk calculator: an ACS-NSQIP resource. HPB (Oxford) 12: 488-497, 2010. PMID: 20815858. DOI: $10.1111 / \mathrm{j} .1477-2574.2010 .00216 . x$

7 Tanaka M, Chari S, Adsay V, Fernandez-del Castillo C, Falconi M, Shimizu M, Yamaguchi K, Yamao K, Matsuno S and International Association of Pancreatology: International consensus guidelines for management of intraductal papillary mucinous neoplasms and mucinous cystic neoplasms of the pancreas. Pancreatology 6: 17-32, 2006. PMID: 16327281. DOI: $10.1159 / 000090023$
8 Tanaka M, Fernández-del Castillo C, Adsay V, Chari S, Falconi M, Jang JY, Kimura W, Levy P, Pitman MB, Schmidt CM, Shimizu M, Wolfgang CL, Yamaguchi K, Yamao K and International Association of Pancreatology: International consensus guidelines 2012 for the management of IPMN and MCN of the pancreas. Pancreatology 12: 183-197, 2012. PMID: 22687371. DOI: 10.1016/j.pan.2012.04.004

9 Tanaka M, Fernández-Del Castillo C, Kamisawa T, Jang JY, Levy P, Ohtsuka T, Salvia R, Shimizu Y, Tada M and Wolfgang CL: Revisions of international consensus Fukuoka guidelines for the management of IPMN of the pancreas. Pancreatology 17: 738753, 2017. PMID: 28735806. DOI: 10.1016/j.pan.2017.07.007

10 Rodriguez JR, Salvia R, Crippa S, Warshaw AL, Bassi C, Falconi M, Thayer SP, Lauwers GY, Capelli P, Mino-Kenudson M, Razo O, McGrath D, Pederzoli P and Fernández-Del Castillo $\mathrm{C}$ : Branch-duct intraductal papillary mucinous neoplasms: observations in 145 patients who underwent resection. Gastroenterology 133: 72-79, 2017. PMID: 17631133. DOI: 10.1053/j.gastro.2007.05.010

11 Jang JY, Park T, Lee S, Kang MJ, Lee SY, Lee KB, Chang YR and Kim SW: Validation of international consensus guidelines for the resection of branch duct-type intraductal papillary mucinous neoplasms. Br J Surg 101: 686-692, 2014. PMID: 24668442. DOI: $10.1002 /$ bjs.9491

12 Facey K, Bradbury I, Laking G and Payne E: Overview of the clinical effectiveness of positron emission tomography imaging in selected cancers. Health Technol Assess 11: iii-iv, xi-267, 2007. PMID: 17999839.

13 Bertagna F, Treglia G, Baiocchi GL and Giubbini R: F18-FDGPET/CT for evaluation of intraductal papillary mucinous neoplasms (IPMN): a review of the literature. Jpn J Radiol 31: 229-236, 2013. PMID: 23315020. DOI: 10.1007/s11604-012-0176-2

14 Sperti C, Bissoli S, Pasquali C, Frison L, Liessi G, Chierichetti F and Pedrazzoli S: 18-fluorodeoxyglucose positron emission tomography enhances computed tomography diagnosis of malignant intraductal papillary mucinous neoplasms of the pancreas. Ann Surg 246: 932-937, 2007. PMID: 18043094. DOI: 10.1097/SLA.0b013e31815c2a29

15 Ohta K, Tanada M, Sugawara Y, Teramoto N and Iguchi H: Usefulness of positron emission tomography (PET)/contrastenhanced computed tomography (CE-CT) in discriminating between malignant and benign intraductal papillary mucinous neoplasms (IPMNs). Pancreatology 17: 911-919, 2017. PMID: 29033011. DOI: 10.1016/j.pan.2017.09.010

16 Roch AM, Barron MR, Tann M, Sandrasegar K, Hannaford KN, Ceppa EP, House MG, Zyromski NJ, Nakeeb A and Schmidt CM: Does PET with CT have clinical utility in the management of patients with intraductal papillary mucinous neoplasm? J Am Coll Surg 221: 48-56, 2015. PMID: 26095551. DOI: 10.1016/j.jamcollsurg.2015.04.020

17 Arima K, Okabe H, Hashimoto D, Chikamoto A, Kuroki H, Taki K, Kaida T, Higashi T, Nitta H, Komohara Y, Beppu T, Takeya $\mathrm{M}$ and Baba H: The neutrophil-to-lymphocyte ratio predicts malignant potential in intraductal papillary mucinous neoplasms. J Gastrointest Surg 19: 2171-2177, 2015. PMID: 26443528. DOI: $10.1007 / \mathrm{s} 11605-015-2973-2$

18 Wilson CB: PET scanning in oncology. Eur J Cancer 28: 508510, 1992. PMID: 1591075.

19 Poeppel TD, Krause BJ, Heusner TA, Boy C, Bockisch A and Antoch G: PET/CT for the staging and follow-up of patients 
with malignancies. Eur J Radiol 70: 382-392, 2009. PMID: 19406595. DOI: 10.1016/j.ejrad.2009.03.051

20 Chikamoto A, Inoue R, Komohara Y, Sakamaki K, Hashimoto D, Shiraishi S, Takamori H, Yamashita YI, Yoshida N, Yamanaka T, Yamashita $\mathrm{Y}$ and Baba $\mathrm{H}$ : Preoperative high maximum standardized uptake value in association with glucose transporter 1 predicts poor prognosis in pancreatic cancer. Ann Surg Oncol 24: 2040-2046, 2017. PMID: 28180987. DOI: 10.1245/s10434-017-5799-1

21 Matsumoto T, Okabe H, Yamashita YI, Yusa T, Itoyama R, Nakao Y, Yamao T, Umzaki N, Tsukamoto M, Kitano Y, Miyata T, Arima K, Nitta H, Hayashi H, Imai K, Chikamoto A and Baba H: Clinical role of fludeoxyglucose (18F) positron emission tomography/computed tomography (18F-FDG PET/CT) in patients with pancreatic neuroendocrine tumors. Surg Today 49: 21-26, 2019. PMID: 30128759 . DOI: 10.1007/s00595-018-1703-2

22 Watanabe A, Harimoto N, Araki K, Yoshizumi T, Arima K, Yamashita Y, Baba H, Tetsuya H, Kuwano H and Shirabe K: A new strategy based on fluorodeoxyglucose-positron emission tomography for managing liver metastasis from colorectal cancer. J Surg Oncol 118: 1088-1095, 2018. PMID: 30261101. DOI: $10.1002 /$ jso. 25250

23 Sultana A, Jackson R, Tim G, Bostock E, Psarelli EE, Cox TF, Sutton R, Ghaneh P, Raraty MG, Neoptolemos JP and Halloran $\mathrm{CM}$ : What is the best way to identify malignant transformation within pancreatic IPMN: A systematic review and meta-analyses. Clin Transl Gastroenterol 6: e130, 2015. PMID: 26658837. DOI: $10.1038 / \operatorname{ctg} .2015 .60$
24 Tanno S, Nakano Y, Sugiyama Y, Nakamura K, Sasajima J, Koizumi K, Yamazaki M, Nishikawa T, Mizukami Y, Yanagawa N, Fujii T, Obara T, Okumura T and Kohgo Y: Incidence of synchronous and metachronous pancreatic carcinoma in 168 patients with branch duct intraductal papillary mucinous neoplasm. Pancreatology 10: 173-178, 2010. PMID: 20484955. DOI: $10.1159 / 000231982$

25 Malleo G, Marchegiani G, Borin A, Capelli P, Accordini F, Butturini G, Pederzoli P, Bassi C and Salvia R: Observational study of the incidence of pancreatic and extrapancreatic malignancies during surveillance of patients with branch-duct intraductal papillary mucinous neoplasm. Ann Surg 261: 984-990, 2015. PMID: 25493361. DOI: 10.1097/SLA.000000000 0000884 26 Marchegiani G, Andrianello S, Borin A, Dal Borgo C, Perri G, Pollini T, Romanò G, D’Onofrio M, Gabbrielli A, Scarpa A, Malleo G, Bassi C and Salvia R: Systematic review, metaanalysis, and a high-volume center experience supporting the new role of mural nodules proposed by the updated 2017 international guidelines on IPMN of the pancreas. Surgery 163: 1272-1279, 2018. PMID: 29454468. DOI: 10.1016/j.surg.2018. 01.009

Received March 14, 2019

Revised March 31, 2019

Accepted April 1, 2019 